\title{
Reflexive orienting to gaze is not luminance dependent
}

\author{
KAITLIN E. W. LAIDLAW \\ University of British Columbia, Vancouver, British Columbia, Canada \\ AND \\ JaY Pratt \\ University of Toronto, Toronto, Ontario, Canada
}

\begin{abstract}
In the investigation of reflexive orienting to cues, two major theories have emerged: One proposes that transients created by the cue trigger attentional shifts, whereas the other argues that object changes are responsible for instigating orienting. In the present study, we examined whether luminance transients produced by the cue can generate reflexive orienting to gaze. Using a temporal order judgment paradigm under luminant or subjectively equiluminant conditions, participants judged which of two peripheral targets onset first. An uninformative gaze cue served to reflexively shift attention toward one object location, thereby temporally prioritizing the target presented there. The results revealed that attention was successfully shifted toward the cued object, as was evidenced by the participants' selecting the cued object as appearing first significantly more often than the uncued object, even when the two onset simultaneously. Critically, the results were comparable across luminance conditions. Our findings reveal that luminance transients are not necessary for triggering orienting to gaze cues. We suggest that the orienting observed here can be better explained via an object-based hypothesis whereby object changes, not transients, trigger reflexive orienting.
\end{abstract}

An object or event that can automatically orient an observer's attention to a peripheral location is known as a reflexive cue (Jonides, 1980; Posner, 1980). Over the years, researchers have sought to determine what cue characteristics are necessary to trigger reflexive orienting. Two general theories have since emerged: the transient hypothesis and the object hypothesis. According to the transient hypothesis, luminance or motion transients serve to signal an important event in the periphery, which in turn triggers the attentional system to shift its focus toward the change (e.g., Franconeri \& Simons, 2003; Jonides \& Yantis, 1988; Yantis \& Jonides, 1984). Alternatively, the object hypothesis states that changes in object properties themselves, and not the transients that often accompany these changes, are responsible for reorienting attention. Object changes such as the abrupt onset of a new object (e.g., Yantis, 1993; Yantis \& Jonides, 1996), the sudden percept of an object from a Gestalt combination of other stimuli (e.g., Kimchi, Yeshurun, \& Cohen-Savransky, 2007; Yeshurun, Kimchi, Sha'shoua, \& Carmel, 2009), or an object suddenly going into motion (e.g., Abrams \& Christ, 2003) have all been shown to elicit involuntary orienting. To elicit reflexive orienting, the factors required by the two hypotheses often coexist (e.g., a new object that creates a change in luminance), but under certain circumstances, the transient and object hypotheses predict very different outcomes.
For example, the object hypothesis predicts that looming stimuli will only attract attention if the onset of motion is perceived, whereas the transient hypothesis is not limited by this constraint (Abrams \& Christ, 2005; Franconeri \& Simons, 2003).

Traditionally, reflexive orienting has been studied using only peripheral cues, but it is now known that centrally presented, uninformative gaze cues also automatically orient attention by pushing attention away from the center and toward a peripheral target (Driver et al., 1999; Friesen \& Kingstone, 2003; Prinzmetal, Leonhardt, \& Garrett, 2008; Stevens, West, Al-Aidroos, Weger, \& Pratt, 2008). Generally, questions concerning how orienting is initiated in response to gaze have been overlooked. Although the object and transient hypotheses were conceptualized to describe orienting produced by peripheral cues, it is possible that attentional cuing by gaze could also be explained by one of these theories. Although Schuller and Rossion (2004) demonstrated that gaze cues do not depend on motion transients to induce orienting, conclusive evidence for or against either theory is limited within the gaze cuing literature. However, luminance transients appear to be critical for accurate perception of gaze direction, and may therefore also play an important role during orienting. For example, it has been demonstrated that reversing the polarity of an image can dramatically reduce the accuracy of

K. E.W. Laidlaw, klaidlaw@psych.ubc.ca 
a participant's judgment of gaze direction (Ricciardelli, Baylis, \& Driver, 2000). This has similarly been shown within a cuing paradigm: When only the eye region of a face is presented in reverse polarity, cuing effects are reversed, such that the observer's attention is pushed in the opposite direction of the gaze (Tipples, 2005). Subtler luminance changes have also been shown to affect perception of gaze direction. For instance, when one side of the sclera is artificially darkened, as might happen if a face were partially in shadow, the apparent direction of gaze is shifted considerably toward the darker side of the sclera (Ando, 2002). Our susceptibility to this illusion can be explained if gaze direction perception is reliant on a luminance-based heuristic, which dictates that the darkest part of the eye does the looking. In support of this, it has been shown that when an image of an eye is observed, simple cells within the striate cortex produce three distinct response amplitudes, which correspond to the pupil and each side of the sclera. When the pupil shifts positions, the relative strengths of the scleral responses change in proportion to the amount of visible area on either side of the pupil (see Langton, Watt, \& Bruce, 2000). Thus, consistent with the transient hypothesis, there is evidence that luminance transients produced when the pupil shifts can instigate an automatic shift of attention toward the perceived direction of gaze.

Although it has been shown that changes in luminance are sufficient to generate orienting to gaze (Tipples, 2005), it is unknown whether luminance transients are necessary to produce an attentional shift in response to gaze. In our study, we address this question by determining whether attentional orienting with gaze cues occurs in the absence of luminance transients. We used a temporal order judgment (TOJ) task, a very sensitive measure of attentional orienting, under luminant and equiluminant conditions to examine the ability of gaze cues to automatically orient attention. To ensure that the equiluminant condition did not contain luminance transients, we had participants complete a variant of the heterochromatic flicker fusion paradigm, which is generally accepted as an appropriate and accurate means of determining subjective equiluminance (e.g., Gunther \& Dobkins, 2002; Lambert, Wells, \& Kean, 2003; Wagner \& Boynton, 1972). During the TOJ task, the participants were asked to judge which of two peripherally presented objects - one cued and one uncued-appeared first. The onset of the two objects was separated by a variable stimulus onset asynchrony (SOA). If a location is cued, it is temporally prioritized, which frequently causes participants to report the cued object as appearing first, even if it is presented a short time after the other object (Shore, Spence, \& Klein, 2001; Stelmach \& Herdman, 1991). Known as the prior entry effect, this phenomenon is a measure of involuntary orienting produced by the cue: Cues that strongly capture attention will produce larger prior entry effects than those that do not. If luminance transients are necessary for reflexive orienting to gaze cues, prior entry effects should be observed only in the luminance condition. Alternatively, if the object hypothesis is correct and object changes within the cue (i.e., the onset or change in position of the pupil within the eye region) are responsible for reflexive orienting, luminant and equiluminant conditions should produce comparable prior entry effects.

\section{METHOD}

\section{Participants}

Fourteen undergraduate students participated in the luminant version of the TOJ (white stimuli on a black background); 12 undergraduates participated in the equiluminant version (green stimuli on a red background). Two participants from each group were removed because of exceptionally poor temporal discrimination at all SOAs, leaving 12 and 10 participants in the luminant and equiluminant conditions, respectively. All of the participants were right-handed and reported normal or corrected-to-normal vision. The mean age was 20.00 years $(S D=2.34)$ for the luminant condition and 21.82 years $(S D=4.17)$ for the equiluminant condition. Seven male participants were tested in the luminant condition, and four male participants were tested in the equiluminant condition.

\section{Apparatus, Stimuli, and Procedure}

The participants were tested using a PC with a VGA monitor and a chinrest. Testing was conducted in a dimly lit, sound-attenuating room.

Before the start of the equiluminant program, each participant performed a heterochromatic flicker fusion task to ensure that the green hue used for the schematic face, placeholders, and target bars was perceptually equiluminant to the red hue chosen for the background (for a similar design, see Wagner \& Boynton, 1972). The participants were presented with a $4^{\circ} \times 4^{\circ}$ square on a black background. The color of the square alternated between red and green at a rate of $30 \mathrm{~Hz}$. When colors with unequal luminosities are alternately presented, the display appears to flicker. However, when colors are adjusted to be perceptually equiluminant to the observer, the flicker disappears. With a standard red color for reference (RGB: $170,0,0 ; 5.28 \mathrm{~cd} / \mathrm{m}^{2}$; CIE coordinates: $x=0.60, y=0.32$ ), the participants increased or decreased the luminance of the green hue using the left and right arrow keys in order to minimize flicker during color alternation. The task was performed eight times, and the initial green hue alternated between very dark (RGB: $0,1,0 ; 0 \mathrm{~cd} / \mathrm{m}^{2}$ ) and very bright (RGB: $0,255,0 ; 48.50 \mathrm{~cd} / \mathrm{m}^{2}$; CIE coordinates: $x=0.30, y=0.60$ ). At the end of the eight trials, an average green value was found for each participant and was entered into the TOJ program to serve as the color of all stimuli. The red value used in the flicker fusion paradigm served as the background color for the TOJ program. The participants in the luminant condition did not complete a luminance-matching task.

Figure 1 shows the sequence of events for a trial. Each trial commenced with a start screen containing a fixation box $\left(0.2^{\circ} \times 0.2^{\circ}\right)$ and two placeholder boxes $\left(2.0^{\circ} \times 2.0^{\circ}, 0.04^{\circ}\right.$ line width $)$, each placed on the horizontal meridian at an $8.0^{\circ}$ eccentricity from the center. After $500 \mathrm{msec}$, a centrally presented schematic face $\left(2.4^{\circ} \times 2.4^{\circ}\right)$ replaced the fixation box and was displayed for $500 \mathrm{msec}$ without pupils, as though the eyes were closed. The pupils $\left(0.4^{\circ} \times 0.4^{\circ}\right)$ were then presented randomly and with equal probability to the left or the right of the eye region. Two hundred milliseconds after pupil onset, a $1.0^{\circ}$ vertical or horizontal bar appeared in the left or right placeholder. The orientation and location of the bar was random, and all combinations were equally probable. A second bar of opposite orientation was presented in the other placeholder after a variable SOA of $0,12,48,84,120$, or $180 \mathrm{msec}$. The bars remained onscreen until a response was made. Using the "F" and "J" keys on the keyboard, the participants input whether the horizontal or vertical bar appeared first. The association between key and bar orientation was counterbalanced across participants, and a sticker showing the correct bar orientation was pasted onto the key for reference. The participants were given 2,000 msec to input their response. A delay of $750 \mathrm{msec}$ separated each trial. 


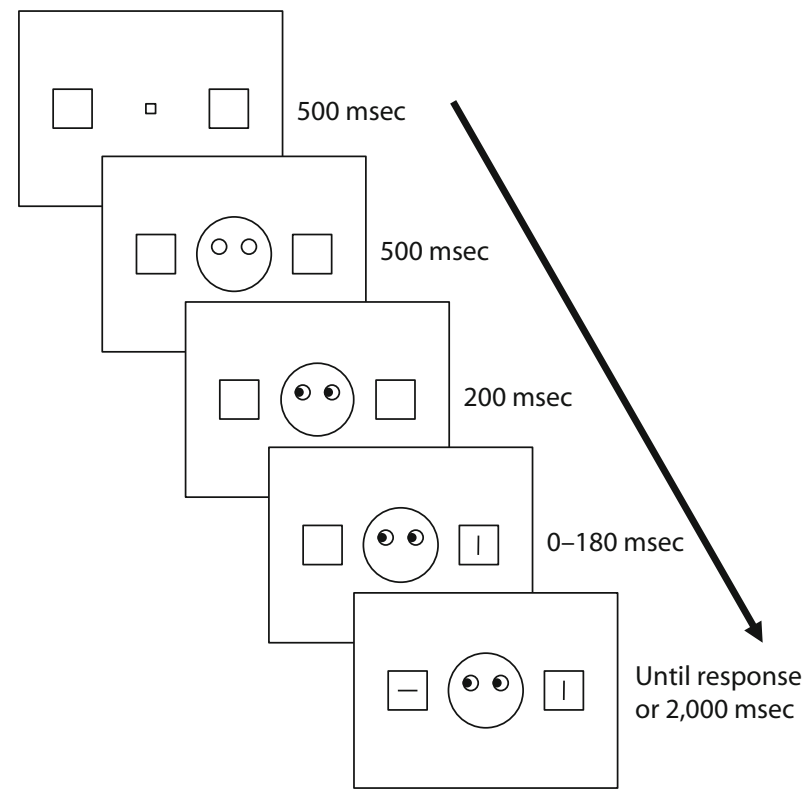

Figure 1. Sample schematic trial sequence for the temporal order judgment task. The stimulus onset asynchrony (SOA) between Target 1 and Target 2 varied randomly among $0,12,48$, 84,120 , and $180 \mathrm{msec}$. The participants pressed a key to indicate which bar orientation appeared first. In the luminant condition, the stimuli (not drawn to scale) were white on a black background; in the equiluminant condition, the stimuli were green on a perceptually equiluminant red background.

Twenty trials for each SOA (6) and cue direction (2) combination were completed, resulting in 240 gaze trials for each luminance condition. The experiment was divided into three blocks, separated by self-paced breaks. The participants completed a minimum of 10 practice trials, until they were comfortable with the association between key and bar orientation.

\section{RESULTS}

The percentage of trials in which the uncued object was reported as appearing before the cued object was calculated for each SOA (Figure 2). The point of subjective simultaneity (PSS) is the point at which participants report the two objects as appearing simultaneously (i.e., the point at which they select the uncued object as appearing first in $50 \%$ of trials). The PSS is calculated as the intercept at the $50 \%$ point of a linear regression line. The participants' individual measures were averaged to obtain an overall PSS. For the luminant condition, the PSS was $57.66 \mathrm{msec}$, and it was $65.97 \mathrm{msec}$ in the equiluminant condition. In other words, the uncued object had to appear an average of 57.66 or $65.97 \mathrm{msec}$ before the cued object for the participant to be unsure of which object onset first. A repeated measures ANOVA was performed with SOA as a within-subjects factor $(0,12,48,84,120$, or $180 \mathrm{msec})$ and luminance as a between-subjects factor (luminant or equiluminant). Nonsphericity was present $\left[\chi^{2}=25.10\right.$, $p=.04]$, and degrees of freedom were adjusted via a Greenhouse-Geisser correction. A main effect of SOA was found $[F(3.44,68.71)=144.81, p<.001]$. Neither the main effect of luminance nor the interaction between SOA and luminance was significant (both $F \mathrm{~s}<1$ ), indicating that luminance did not significantly influence the magnitude of prior entry.

\section{DISCUSSION}

It is known that gaze can automatically cue attention (Driver et al., 1999; Friesen \& Kingstone, 2003; Prinzmetal et al., 2008; Stevens et al., 2008), but few researchers have investigated the mechanisms by which gaze is capable of producing the necessary attentional shift to

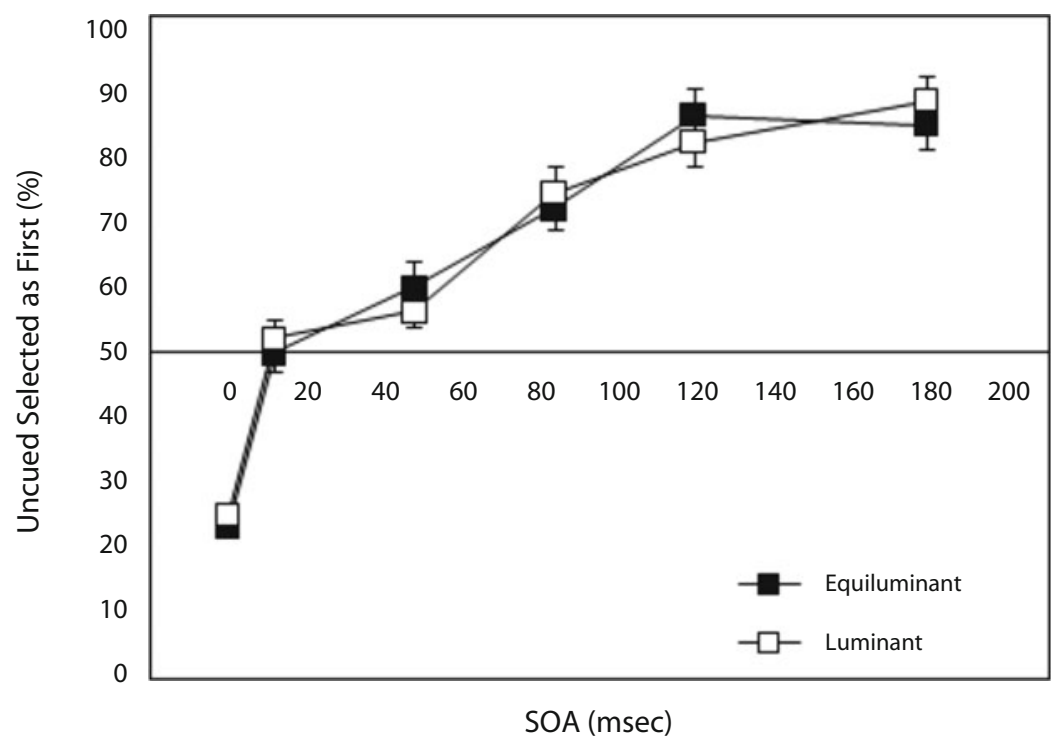

Figure 2. Mean percentage of trials in which the uncued target was perceived as appearing first when it objectively onset before the cued target, at all stimulus onset asynchronies (SOAs) in the luminant and equiluminant conditions. Error bars denote standard errors. 
the gazed-at location. In the peripheral cue literature, evidence has accumulated in support of two distinct theories put forth to explain how reflexive orienting is initiatedthe transient hypothesis and the object hypothesis-but few researchers have extended these theories to gaze cues. Research indicates that luminance transients are critical for accurate gaze direction perception, but until now, it was unknown whether the process of orienting to gaze is also dependent on luminance changes. To determine whether gaze cuing can be explained via the transient hypothesis, we compared involuntary attentional orienting produced during luminant and equiluminant conditions. Our results show that gaze cues do not require luminance transients in order to produce significant reflexive orienting. When the cued and uncued target bars were presented simultaneously, the cued object was reported as appearing first significantly more often than the uncued object. Although the cue was unpredictive of which target would onset first, robust visual prior entry effects were found for both luminant and equiluminant conditions. These results provide strong evidence against the luminance transient hypothesis.

Our findings demonstrate that luminance is not necessary to trigger a reflexive attentional shift toward a cued location. Although previous research using peripheral cues has revealed that luminance can influence orienting, we found no evidence of this when gaze was tested. The unique attentional pathways used by these different types of cues may provide an explanation for this potential discrepancy. Although traditional exogenous cues appear to rely on subcortical orienting mechanisms (Reuter-Lorenz, Hughes, \& Fendrich, 1991; Robinson \& Kertzman, 1995), gaze orienting may also depend on cortical processes (Kingstone, Friesen, \& Gazzaniga, 2000). If peripheral cues bypass cortical involvement during attentional shifting, orienting may be vulnerable to low-level stimulus transients, such as those produced by luminance. In contrast, higher level cortical areas, possibly located within the frontal lobe (Vecera \& Rizzo, 2006), may serve a mediating role during gaze cuing, thus reducing gaze's susceptibility to irrelevant luminance changes. Such a mediation process would be especially important in everyday life; otherwise, the simplest events, such as having a face partially in shadow, would significantly hamper the usefulness of gaze as an attentional cue. Note that this account blurs the classic notions of endogenous and exogenous orienting, since gaze cues appear to trigger involuntary shifts of attention that result from the top-down processing of gaze information.

Luminance is only one type of transient, and although we have shown that transients of this type are unnecessary for orienting to gaze, other transients may be important. For example, some motion transients have been hypothesized to be important in attentional capture to peripheral objects (e.g., Franconeri \& Simons, 2003). In contrast, however, cuing effects to gaze are observed even when motion transients are controlled for, either by presenting a static face with gaze already shifted (Schuller \& Rossion, 2004) or by keeping the pupil static and shifting the head in the opposite direction of the gaze shift (Bayliss, di Pellegrino, \& Tipper, 2005). In the present study, we controlled for motion transients by presenting the pupil already displaced to the left or right of the sclera, and as such, we do not believe that motion transients were responsible for the orienting observed. It remains possible that color transients may play a role in shifting attention, but we cannot speak to this possibility, since our equiluminant condition necessitated a color display. Although the transient hypothesis has yet to be ruled out in its entirety, several of its components can be, which suggests that an alternative hypothesis may be better suited for explaining how orienting to gaze is accomplished.

Given the evidence against the necessity of luminance and motion transients to initiate orienting to gaze cues, we propose that the object hypothesis provides a more parsimonious account of how gaze reflexively orients attention. Ando (2002) suggested that geometric cues from the eye may be critical for discerning gaze direction; this, too, may be used to trigger orienting. When a change is made within an object, attention is shifted automatically to that change. With a peripheral cue, an object change will draw attention reflexively toward the object. With gaze, the shift of the pupil's position changes the geometry of the eye region, which may initially attract attention to the eyes itself. When the aforementioned unique cortical processes come online, however, they may instigate a rapid shift of attention to the periphery, where gaze is directed. Lobmaier, Fischer, and Schwaninger (2006) demonstrated that we attribute intention to gaze, such that if an object is in the periphery and gaze is directed roughly toward that object, we will infer that the gaze is intended to reference that object. As such, it is possible that our attention is involuntarily shifted away from the object change within the eye and directed toward the periphery, where we anticipate the gazer's goal to appear. On the basis of the present findings, our attribution of intention to gaze is reliant on changes perceived within the eye itself and not from any luminance transients associated with these changes.

\section{AUTHOR NOTE}

This work was supported by an NSERC grant to J.P. The authors thank Ruo Gu Muo for programming the flicker fusion task. Correspondence concerning this article should be addressed to K. E. W. Laidlaw, Psychology Department, University of British Columbia, Vancouver, BC, V6T 1Z4 Canada (e-mail: klaidlaw@psych.ubc.ca).

\section{REFERENCES}

Abrams, R. A., \& Christ, S. E. (2003). Motion onset captures attention. Psychological Science, 14, 427-432.

Abrams, R. A., \& Christ, S. E. (2005). Onset but not offset of irrelevant motion disrupts inhibition of return. Perception \& Psychophysics, 67, 1460-1467.

ANDO, S. (2002). Luminance-induced shift in the apparent direction of gaze. Perception, 31, 657-674.

Bayliss, A. P., Di Pellegrino, G., \& Tipper, S. P. (2005). Sex differences in eye gaze and symbolic cuing of attention. Quarterly Journal of Experimental Psychology, 58A, 631-650.

Driver, J., Davis, G., Ricciardelli, P., Kidd, P., Maxwell, E., \& BARON-CoHEN, S. (1999). Gaze perception triggers visuospatial orienting by adults in a reflexive manner. Visual Cognition, 6, 509-540.

Franconeri, S. L., \& SimONS, D. J. (2003). Moving and looming stimuli capture attention. Perception \& Psychophysics, 65, 999-1010.

Friesen, C. K., \& Kingstone, A. (2003). Abrupt onsets and gaze direc- 
tion cues trigger independent reflexive attentional effects. Cognition, 87, B1-B10.

Gunther, K. L., \& Dobkins, K. R. (2002). Individual differences in chromatic (red/green) contrast sensitivity are constrained by the relative number of L- versus M-cones in the eye. Vision Research, $\mathbf{4 2}$, $1367-1378$

Jonides, J. (1980). Toward a model of the mind's eye's movement. Canadian Journal of Psychology, 34, 103-112.

Jonides, J., \& YANTIS, S. (1988). Uniqueness of abrupt visual onset in capturing attention. Perception \& Psychophysics, 43, 346-354.

Kimchi, R., Yeshurun, Y., \& Cohen-Savransky, A. (2007). Automatic, stimulus-driven attentional capture by objecthood. Psychonomic Bulletin \& Review, 14, 166-172.

Kingstone, A., Friesen, C. K., \& Gazzaniga, M. (2000). Reflexive joint attention depends on lateralized cortical connections. Psychological Science, 11, 159-166.

Lambert, A., Wells, I., \& Kean, M. (2003). Do isoluminant color changes capture attention? Perception \& Psychophysics, 65, 495-507.

Langton, S. R. H., Watt, R. J., \& Bruce, V. (2000). Do the eyes have it? Cues to the direction of social attention. Trends in Cognitive Sciences, 4, 50-59.

Lobmaier, J. S., Fischer, M. H., \& Schwaninger, A. (2006). Objects capture perceived gaze direction. Experimental Psychology, 53, 117122.

Posner, M. I. (1980). Orienting of attention. Quarterly Journal of Experimental Psychology, 32, 3-25.

Prinzmetal, W., Leonhardt, J., \& Garrett, R. (2008). Does gaze direction affect accuracy? Visual Cognition, 16, 567-584

Reuter-Lorenz, P. A., Hughes, H. C., \& Fendrich, R. (1991). The reduction of saccadic latency by prior offset of the fixation point: An analysis of the gap effect. Perception \& Psychophysics, 49, 167-175.

Ricciardelli, P., BAYLIS, G., \& DRIVER, J. (2000). The positive and negative of human expertise in gaze perception. Cognition, 77, B1-B14.

Robinson, D. L., \& Kertzman, C. (1995). Covert orienting of attention in macaques: III. Contributions of the superior colliculus. Journal of Neurophysiology, 74, 713-721.
Schuller, A.-M., \& Rossion, B. (2004). Perception of static eye gaze direction facilitates subsequent early visual processing. Clinical Neuropsychology, 115, 1161-1168.

Shore, D. I., Spence, C., \& Klein, R. M. (2001). Visual prior entry. Psychological Science, 12, 205-212.

Stelmach, L. B., \& Herdman, C. M. (1991). Directed attention and perception of temporal order. Journal of Experimental Psychology: Human Perception \& Performance, 17, 539-550.

Stevens, S. A., West, G. L., Al-Aidroos, N., Weger, U. W., \& Pratt, J. (2008). Testing whether gaze cues and arrow cues produce reflexive or volitional shifts of attention. Psychonomic Bulletin \& Review, 15, 1148-1153.

TipPLeS, J. (2005). Orienting to eye gaze and face processing. Journal of Experimental Psychology: Human Perception \& Performance, 31, 843-856.

VeCERA, S. P., \& Rizzo, M. (2006). Eye gaze does not produce reflexive shifts of attention: Evidence from frontal-lobe damage. Neuropsychologia, 44, 150-159.

WAGNER, G., \& Boynton, R. M. (1972). A comparison of four methods of heterochromatic photometry. Journal of the Optical Society of America, 62, 1508-1515.

YANTIS, S. (1993). Stimulus-driven attentional capture and attentional control settings. Journal of Experimental Psychology: Human Perception \& Performance, 19, 676-681.

YANTIS, S., \& Jonides, J. (1984). Abrupt visual onsets and selective attention: Evidence from visual search. Journal of Experimental Psychology: Human Perception \& Performance, 10, 601-621.

YANTIS, S., \& Jonides, J. (1996). Attentional capture by abrupt onsets: New perceptual objects or visual masking? Journal of Experimental Psychology: Human Perception \& Performance, 22, 1505-1513.

Yeshurun, Y., Kimchi, R., Sha'shoua, G., \& Carmel, T. (2009). Perceptual objects capture attention. Vision Research, 49, 1329-1335.

(Manuscript received June 10, 2009;

revision accepted for publication August 11, 2009.) 